\title{
A NECESSARY CONDITION FOR A NON-NEGATIVE SOLUTION OF AN INTEGRAL EQUATION
}

\author{
T.A. BURTON
}

Communicated by Neville Ford

\begin{abstract}
One of the cornerstones of the theory of linear integral equations is the establishment of properties of the resolvent kernel. This theory has consequences in the study of a wide class of nonlinear integral equations, including those of the form
\end{abstract}

$$
x(t)=f(t)-\int_{0}^{t} C(t-s) h(s, x(s)) d s,
$$

that commonly arise in applied mathematics. Using such a theory, in this work, we study conditions on (1) that relate to the existence of a solution $x$ that is non-negative on $[0, \infty)$. In the classical literature, one finds amongst sufficient conditions for non-negativity of $x$ on $[0, \infty)$, the requirements

$f(t)>0$ on $[0, \infty), C(t)>0$ on $(0, \infty)$, and $\frac{f(T)}{f(t)} \leq \frac{C(T-s)}{C(t-s)}$

when $0 \leq s<T<t$, for $t \in[0, \infty)$. Our work begins here when we show that this assumption implies that $\int_{0}^{\infty} f(t) d t=\infty$ whenever $\int_{0}^{\infty} C(t) d t=\infty$. This motivated the conjecture that, when $\int_{0}^{\infty} C(t) d t=\infty$, then a necessary condition for the existence of a non-negative solution is that $\int_{0}^{\infty} f(t) d t=\infty$. The proof of that conjecture is the main result of this paper.

Finally, we point out that, while (1) seems very special and possibly of narrow interest, that is not the case. Equation (1) is of prime importance in establishing properties of the resolvent kernel for scalar equations arising in mathematical physics from areas as diverse as heat problems and problems in turbulence as well as fractional differential equations of both Caputo and Riemann-Liouville type. There is a myriad of real-world problems modeled by the latter two types, and such studies are among the very active in mathematics today.

2010 AMS Mathematics subject classification. Primary 45A05, 45D05, 45 G05. Keywords and phrases. Integral equations, positive solutions.

Received by the editors on October 3, 2014, and in revised form on April 19, 2015. 
1. Introduction. The purpose of this paper is to show that one set of classical conditions ensuring the existence of a non-negative solution of an important integral equation contains a condition which is also necessary. In a great many cases that necessary condition is so easy to check that it can prevent the investigator from spending much time and effort attempting to prove something that is not true. It can also be a guide in constructing mathematical models.

Miller [3, pages 210-224] presents a very complete discussion of the properties of the resolvent kernel, $R$, for an integral equation with kernel, $C$, of convolution type. He formulates conditions on $C$ which are compatible with a vast collection of real-world problems and which yield a positive resolvent. While the equation for $R$ is linear, Miller chooses to do more and seeks a non-negative solution on $[0, \infty)$ of the scalar equation

$$
x(t)=f(t)-\int_{0}^{t} C(t-s) h(s, x(s)) d s
$$

when $f, C$, and $h$ are continuous with

$$
\begin{aligned}
& f:[0, \infty) \longrightarrow(0, \infty), \quad C:(0, \infty) \longrightarrow(0, \infty), \\
& h:[0, \infty) \times \Re \longrightarrow \Re, \quad x \neq 0 \Longrightarrow x h(t, x)>0 .
\end{aligned}
$$

Conditions $(\mathrm{C} 1),(\mathrm{C} 2)$ and $(\mathrm{C} 3)$ below will generate a continuous resolvent, $R:(0, \infty) \rightarrow \Re$, and, when $\int_{0}^{\infty} C(t) d t=\infty$, then it will satisfy

$$
0 \leq R(t) \leq C(t), \quad 0 \leq t<\infty, \quad \int_{0}^{\infty} R(t) d t=1 .
$$

Moreover, when $C$ is completely monotone

$$
(-1)^{k} C^{(k)}(t) \geq 0 \text { for } k=0,1,2, \ldots, \text { and } 0<t<\infty,
$$

then the same is true for $R$, and it is also true that $R>0$.

Those three conditions are:

C1 $C:(0, \infty) \rightarrow(0, \infty)$ is continuous and is in $L^{1}(0,1)$.

C2 $C(t)$ is positive and non-increasing for $t>0$.

C3 For each $T>0$, the function $C(t) / C(t+T)$ is non-increasing in $t$ for $0<t<\infty$. 
These conditions are satisfied, for example, for all kernels of the form $t^{q-1}, 0<q<1$, found in fractional differential equations of both Caputo and Riemann-Liouville type as well as a wide range of problems from mathematical physics such as turbulence to heat conduction.

Miller's first step in studying the resolvent for the kernel $C$ is to prove the following theorem.

Theorem 1.1. Consider the real scalar equation (1) in which $f$ is continuous and positive for $t \in[0, \infty)$. Let $C$ be positive, continuous and locally $L^{1}$ for $0<t<\infty$. Suppose that $h$ is measurable in $(t, x)$ and continuous in $x$ for $t \geq 0$ and $x \in \Re$ with $x h(t, x) \geq 0$ for all $(t, x)$. If

$$
f(T) / f(t) \leq C(T-s) / C(t-s)
$$

whenever $0 \leq s<T<t$, then (1) has a solution which satisfies $0 \leq x(t) \leq f(t)$ for all $t \geq 0$.

In our work, we require strict inequality $x h(t, x)>0$ for $x \neq 0$. Obviously, if $h(t, x) \equiv 0$ then $x(t)=f(t)$ is a positive solution.

Pointwise conditions of form (3) are very precise but can be difficult to consolidate into a readily grasped principle. Our first result will extract a simple principle from (3) and leads us to the main result, Theorem 3.1.

Theorem 1.2. Let the continuity conditions with (1) as well as (3) hold with $f$ and $C$ positive. Fix $0 \leq s<T$, and take $t>T$. If $C \in L^{1}(0,1]$, and if $\int_{0}^{\infty} C(u) d u=\infty$, then:

$$
\int_{0}^{\infty} f(s) d s=\infty
$$

and

$$
\frac{\int_{T}^{t} f(u) d u}{\int_{T-s}^{t-s} C(v) d v}
$$

is bounded strictly away from zero as $t \rightarrow \infty$. 
Proof. Fix $0 \leq s<T$, and integrate

$$
C(t-s) \leq \frac{C(T-s)}{f(T)} f(t)
$$

for $T<t$, obtaining

$$
\int_{T}^{t} C(u-s) d u \leq \frac{C(T-s)}{f(T)} \int_{T}^{t} f(u) d u
$$

so that

$$
\begin{aligned}
\int_{T-s}^{t-s} C(v) d v & \leq \frac{C(T-s)}{f(T)} \int_{T}^{t} f(u) d u \\
& <\frac{C(T-s)}{f(T)} \int_{T}^{\infty} f(u) d u,
\end{aligned}
$$

showing that the integral of $f$ also diverges. The top line in (3a) yields (ii).

This result represents an average and, by contrast, the inequality in (3) must hold for every set of values $0 \leq s<T<t$. Generally, (3) demands that $f$ not suddenly decrease faster than $C$ and that is nicely shown in the first inequality in (3a). We will be mainly interested here in (i), but (ii) is also very handy. Notice that (ii) offers a test showing that (3) fails if $f(t)=1 /(t+1)$ and $C(t)=t^{q-1}, \quad 0<q<1$.

Central idea of this paper. For non-negative kernels with infinite integral, (3a) is one part of the sufficient conditions for a non-negative solution and this requires that $f$ have an infinite integral. We study kernels satisfying $(\mathrm{C} 1),(\mathrm{C} 2)$ and $(\mathrm{C} 3)$ with infinite integrals and show that the infinite integral of $f$ is also a necessary condition for a nonnegative solution.

2. A transformation. It is obvious that there will be a nonnegative solution of (1) just in case $f(t)$ is never exceeded by the integral in (1). When we assume that $C$ has infinite integral, then $C$ acts as a weight function on $h(t, x(t))$ creating difficulties in determining which term on the right-hand-side of (1) dominates the other. It turns out that there is a known transformation which will allow us to make this required comparison between the terms in (1). 
This transformation was derived in [1], which contains a complete list of references. When $C$ in (1) satisfies (C1)-(C3), with $\int_{0}^{\infty} C(t) d t=$ 1 , then there is a transformation which yields an equivalent integral equation having a kernel, $R$, satisfying (2).

Assume (C1)-(C3). Begin with (1) and add and subtract $x(s)$ in the integrand, obtaining

$$
x(t)=f(t)-\int_{0}^{t} C(t-s) x(s) d s+\int_{0}^{t} C(t-s)[x(s)-h(s, x(s))] d s .
$$

For the linear part,

$$
z(t)=f(t)-\int_{0}^{t} C(t-s) z(s) d s
$$

there is the resolvent equation

$$
R(t)=C(t)-\int_{0}^{t} C(t-s) R(s) d s,
$$

where $R$ satisfies

$$
0 \leq R(t) \leq C(t)
$$

for all $t>0$. Now we have

$$
\int_{0}^{\infty} C(s) d s=\infty \Longrightarrow \int_{0}^{\infty} R(s) d s=1
$$

while

$$
\int_{0}^{\infty} C(s) d s=\alpha<\infty \Longrightarrow \int_{0}^{\infty} R(s) d s=\frac{\alpha}{1+\alpha} .
$$

We will only be considering the first case. Among its many uses, the resolvent is used to write

$$
z(t)=f(t)-\int_{0}^{t} R(t-s) f(s) d s
$$

Finally, Miller [3, pages 167-193] develops a variation of parameters formula under conditions admitting interchange of order of integration in the form

$$
x(t)=z(t)+\int_{0}^{t} R(t-s)[x(s)-h(s, x(s))] d s .
$$


Strategy. Our task is to arrange matters so that, if $x(t)$ is a nonnegative solution of (7), then, for large $t$, we obtain

$$
\begin{aligned}
(1 / 2) \int_{0}^{t / 2} h(s, x(s)) d s & \leq \int_{0}^{t} z(s) d s \\
& =\int_{0}^{t}\left[f(u)-\int_{0}^{u} R(u-s) f(s) d s\right] d s .
\end{aligned}
$$

Here it is critical that $(\mathrm{C} 1)-(\mathrm{C} 3)$ hold and that $\int_{0}^{\infty} C(t) d t=\infty$ so that $\int_{0}^{\infty} R(s) d s=1$. In that case, this displayed relation will imply

$$
(1 / 2) \int_{0}^{t / 2} h(s, x(s)) d s \leq \int_{0}^{t} \int_{t-s}^{\infty} R(v) d v f(s) d s .
$$

If $f \in L^{1}[0, \infty)$, then the right-hand-side is the convolution of an $L^{1}$ function with a bounded continuous function tending to zero, and we see that it tends to zero. This is a contradiction to the left-hand-side being bounded below by a positive constant.

3. (C1)-(C3) hold and $\int_{0}^{\infty} C(s) d s=\infty$. In the proof below, we will use a relation from [2] that, if $G$ is continuous on $[0, \infty)$ and if $T$ is so large that $\int_{0}^{T / 2} R(s) d s>1 / 2$, then

$$
(1 / 2) \int_{0}^{T / 2}|G(s)| d s \leq \int_{0}^{T} \int_{0}^{u} R(u-s)|G(s)| d s d u .
$$

Also, we will frequently interchange the order of integration which is allowed because $f, R$ and $h(t, x(t))$ are absolutely integrable on the intervals involved in that interchange.

Theorem 3.1. Assume that:

(a) (C1)-(C3) hold, $\int_{0}^{\infty} R(s) d s=1$, and $R$ is the resolvent of $C$,

(b) $h:[0, \infty) \times \Re \rightarrow \Re$ is continuous and $h(t, x)>0$ if $x>0$,

(c) $f:[0, \infty) \rightarrow(0, \infty)$ is continuous and $\int_{0}^{\infty} f(s) d s<\infty$.

Then

$$
x(t)=f(t)-\int_{0}^{t} R(t-s) f(s) d s+\int_{0}^{t} R(t-s)[x(s)-h(s, x(s))] d s
$$

does not have a non-negative solution on $[0, \infty)$. 
Proof. Assume, by way of contradiction, that there is a solution, $x$, on $[0, \infty)$ with $x(t) \geq 0$. As usual, denote the first two terms on the right in the display by $z(t)$. We can integrate the equation and have

$$
\begin{aligned}
\int_{0}^{t} x(s) d s= & \int_{0}^{t} z(s) d s+\int_{0}^{t} \int_{0}^{u} R(u-s)[x(s)-h(s, x(s))] d s \\
= & \int_{0}^{t} z(s) d s+\int_{0}^{t} \int_{s}^{t} R(u-s) x(s) d u d s \\
& -\int_{0}^{t} \int_{0}^{u} R(u-s) h(s, x(s)) d s d u \\
\leq & \int_{0}^{t} z(s) d s+\int_{0}^{t} x(s) d s \\
& -\int_{0}^{t} \int_{0}^{u} R(u-s) h(s, x(s)) d s d u .
\end{aligned}
$$

Cancel the like terms on the left and right sides. We will suppose that $t$ is so large that $\int_{0}^{t / 2} R(u) d u>1 / 2$ and obtain

$$
\begin{aligned}
\frac{1}{2} \int_{0}^{t / 2} h(s, x(s)) d s & \leq \int_{0}^{t} \int_{0}^{u} R(u-s) h(s, x(s)) d s d u \\
& \leq \int_{0}^{t} z(s) d s .
\end{aligned}
$$

We will show that the right-hand-side tends to zero but the left does not. Recall that $f \in L^{1}[0, \infty)$, and write that last term as

$$
\begin{aligned}
& \int_{0}^{t}\left[f(u)-\int_{0}^{u} R(u-s) f(s) d s\right] d u \\
& =\int_{0}^{t} f(u) d u-\int_{0}^{t} \int_{s}^{t} R(u-s) d u f(s) d s \\
& =\int_{0}^{t} f(u) d u-\int_{0}^{t} \int_{0}^{t-s} R(v) d v f(s) d s \\
& =\int_{0}^{t} f(u)\left[1-\int_{0}^{t-u} R(v) d v\right] d u \\
& =\int_{0}^{t} f(u) \int_{t-u}^{\infty} R(v) d v d u .
\end{aligned}
$$


The last term tends to zero since it is the convolution of a continuous $L^{1}$ function with a bounded continuous function tending to zero. Note that $x, f$ and $h(t, x(t))$ are continuous, while $C \in L^{1}(0,1]$ and $f(0)>0$. From this, it follows that $x(0)=f(0)>0$ so $x$ and $h(t, x(t))$ are both positive on some interval $[0, L]$ with $L>0$. This means that

$$
\int_{0}^{t / 2} h(s, x(s)) d s
$$

is bounded below by some positive number as $t \rightarrow \infty$. This is a contradiction to the right-hand-term of $(*)$ tending to zero.

\section{REFERENCES}

1. T.A. Burton, Fractional differential equations and Lyapunov functionals, Nonlin. Anal.: TMA 74 (2011), 5648-5662.

2. T.A. Burton and Bo Zhang, $L^{p}$-solutions of fractional differential equations, Nonlin. Stud. 19 (2012), 307-324.

3. R.K. Miller, Nonlinear Volterra integral equations, Benjamin, Menlo Park, CA, 1971.

Northwest Research Institute, 732 Caroline St., Port Angeles, Wa 98362 Email address: taburton@olypen.com 